\title{
The Intrinsic Properties of Curves
}

\author{
By Professor M. T. Naraniengar
}

(Read 8th May, 1908)

Any property of a curve which depends solely upon its form may be styled intrinsic. Thus the circle of curvature at a point, the relation between $s$ and $\psi$, the envelope of the normals, the envelope of straight lines making a constant angle with the curve, etc., are all intrinsic properties. It is proposed to investigate a few of these properties in this paper.*

\section{I.}

(1) Let a straight line $P Q$ make a constant angle $\lambda$ with a given curve at any point $P$; then the envelope of $P Q$ may be geometrically found as follows.

. If PC, $\mathrm{P}^{\prime} \mathrm{C}$ (Fig. 17) be consecutive normals to the given curve, and $P Q, P^{\prime} Q$ consecutive positions of the straight line making a constant angle with the curve, then evidently TPCQP' is a circle. Therefore $\angle \mathrm{CQT}$ is right and ultimately $\angle \mathrm{CQP}$ is a right angle. Hence the envelope touches the straight line at the foot of the perpendicular from $\mathrm{C}$, the centre of curvature. Also $P Q=\rho \sin \lambda$, where $\rho$ is the rudius of curvature at $P$.

(2) Again, if $Q^{\prime}$ be a neighbouring point on the envelope,

$$
\mathbf{Q Q} \mathbf{Q}^{\prime}=\mathbf{P}^{\prime} \mathbf{Q}^{\prime}-\mathbf{P Q}+\mathbf{P P}^{\prime} \cos \lambda,
$$

i.e., $\quad \delta s^{\prime}=(\rho+\delta \rho) \sin \lambda-\rho \sin \lambda+\delta s \cos \lambda=\delta \rho \sin \lambda+\delta s \cos \lambda$

the accented letters referring to the locus of $Q$.

Also $\delta \psi^{\prime}=\delta \psi$, since $\psi^{\prime}-\psi=\lambda$

$$
\begin{gathered}
\therefore \frac{\delta s^{\prime}}{\delta \psi^{\prime}}=\frac{\delta \rho}{\delta \psi} \sin \lambda+\frac{\delta s}{\delta \psi} \cos \lambda \\
\therefore \rho^{\prime} \text { or } \frac{d s^{\prime}}{d \psi^{\prime}}=\rho_{1} \sin \lambda+\rho \cos \lambda, \text { where } \rho_{1}=\frac{d \rho}{d \psi}
\end{gathered}
$$

which is equal to the radius of curvature of the evolute.

* [Full references to the literature of the problem dealt with in I. will be found in Loria, spezielle algebraische und transscendente ebene Kurven. Leipzig, 1902, p. 626.

ED. E.M.S.P.] 
(3) Let $\mathbf{E}$ be the centre of curvature of the evolute; then, if $\mathrm{EN}$ be perpendicular to $\mathrm{QC}, \mathrm{QN}=\rho \cos \lambda+\rho_{1} \sin \lambda$, so that $\mathrm{N}$ is the centre of curvature of the locus of $Q$. In other words, the centre of curvature at $Q$ is the projection of the centre of curvature of the evolute on the normal at $Q$; and it is related to the evolute just as $Q$ is related to the original curve.

(4) Further, since $\delta s^{\prime}=\delta \rho \sin \lambda+\delta s \cos \lambda$, by integrating we have $s^{\prime}=\rho \sin \lambda+s \cos \lambda+a$ constant. From this the intrinsic equation to the envelope of $P Q$ may be expressed.

(5) From the relation $\rho^{\prime}=\rho_{1} \sin \lambda+\rho \cos \lambda_{1}$ we can deduce that

$$
\begin{aligned}
& \text { (i) } \frac{d \rho^{\prime}}{d \psi^{\prime}}=\rho_{2} \sin \lambda+\rho_{1} \cos \lambda \\
& \text { (ii) } \frac{d^{2} \rho^{\prime}}{d \psi^{\prime 2}}=\rho_{3} \sin \lambda+\rho_{2} \cos \lambda
\end{aligned}
$$

and so on; which means that the centre of curvature of the evolute of $Q$ is the projection of the centre of curvature of the second evolute of the original curve, and so on.

(6) The locus of $Q$ may be regarded as the involute of a curve similarly related to the evolute of the original. Also a system of parallel curves $(P)$ will have a system of parallel curves, $(Q)$, whose evolutes, viz., (C) and (N) are similarly related.

These considerations show that the locus of $\mathrm{N}$ may be styled an oblique $(\lambda)$ evolute of the original curve $P$.

\section{II.}

(1) Let $P Q$ be the diameter of curvature at $P$ and $C D$ that of the evolute, then the locus of $Q$ will have $Q D$ for normal.

For if $P Q, P^{\prime} Q^{\prime}$ (Fig. 18) be consecutive positions of the diameter, then $\mathrm{PQ}=2 \rho$ and $\mathrm{P}^{\prime} \mathrm{Q}^{\prime}=2(\rho+\delta \rho)$. By projection we have in the limit

$$
\tan \phi=\mathrm{L} \frac{\mathbf{Q R}}{\mathbf{Q}^{\prime} \mathbf{R}}=\mathbf{L} \frac{\rho \delta \psi}{2 \delta \rho}=\frac{\rho}{2 \rho_{1}}
$$

Hence $\phi=\angle Q D C$, that is $D Q$ is the normal at $Q$. 
(2) If $Q Q^{\prime}$ be denoted by $\delta s^{\prime}$,

$$
\begin{gathered}
\left(\delta \gamma^{\prime}\right)^{2}=\mathrm{QR}^{2}+\mathrm{Q}^{\prime} \mathrm{R}^{2} \\
\therefore\left(\frac{\delta s^{\prime}}{\delta \psi}\right)^{2}=\left(\frac{\delta s}{\delta \psi}\right)^{2}+4\left(\frac{\delta \rho}{\delta \psi}\right)^{2} \text { and } \therefore\left(\frac{d s^{\prime}}{d \psi}\right)^{2}=\rho^{2}+4 \rho_{\mathrm{j}}{ }^{2}=\mathrm{QD}^{2},
\end{gathered}
$$

whence

$$
\frac{d s^{\prime}}{d \psi}=\mathrm{QD}
$$

Also if the tangents at $\mathrm{Q}$ and $\mathrm{P}$ make angles $\psi^{\prime}$ and $\psi$ with a fixed straight line,

$$
\psi=\psi^{\prime}+\frac{\pi}{2}-\phi
$$

and therefore

$$
\frac{d \psi^{\prime}}{d \psi}=1+\frac{d \phi}{d \psi} . \quad \text { But } \sec ^{2} \phi \frac{d \phi}{d \psi}=\frac{\rho_{1}{ }^{2}-\rho \rho_{2}}{2 \rho_{2}{ }^{2}},
$$

therefore

$$
\frac{d \psi^{\prime}}{d \psi}=1+\frac{2\left(\rho_{1}^{2}-\rho \rho_{2}\right)}{4 \rho_{1}^{2}+\rho^{2}}
$$

Hence $\quad \rho^{\prime}=\frac{d s^{\prime}}{d \psi^{\prime}}=\frac{d s^{\prime}}{d \psi} / \frac{d \psi^{\prime}}{d \psi}=\frac{\left(\rho^{2}+4 \rho_{1}^{2}\right)^{2}}{\rho^{2}+6 \rho_{1}^{2}-\rho \rho_{2}}$.

(3) The centre of curvature at $Q$ may be geometrically determined as follows.

Differentiating $\quad 2 \rho_{1} \sin \phi-\rho \cos \phi=0$

we get

$$
\frac{d \phi}{d \psi}=\frac{\rho_{1} \cos \phi-2 \rho_{2} \sin \phi}{2 \rho_{1} \cos \phi+\rho \sin \phi}
$$

Now, if EF be the diameter of curvature at $\mathbf{E}$ of the second evolute and $\mathrm{H}$ the projection of $\mathrm{F}$ on $\mathrm{QD}$.

$$
\frac{d \phi}{d \psi}=-\frac{\mathrm{HD}}{\mathrm{QD}} \text { and therefore } \frac{d \psi^{\prime}}{d \psi}=1-\frac{\mathrm{DH}}{\mathrm{TD}}=\frac{\mathrm{TH}}{\mathrm{TD}}
$$

Hence $\rho^{\prime}=Q^{2} / \mathrm{TH}$, since $\mathrm{QD}=\mathrm{TD}$. 


\section{III.}

More generally, let $\mathrm{PQ}$ be the chord of curvature subtending a constant angle $2 \lambda$ at the centre, then the properties of the locus of $\mathbf{Q}$ may be similarly determined.

1. $P Q, P^{\prime} Q^{\prime}$ (Fig. 19) being consecutive positions, it is obvious that they intersect at $\mathrm{N}$ so that $\mathrm{C}, \mathrm{N}, \mathrm{P}, \mathrm{P}^{\prime}$ are cyclic, and therefore $\mathrm{CN}$ is ultimately perpendicular to $\mathrm{PN}$.

If $P Q$ be projected on $P^{\prime} Q^{\prime}$, we see that, in the limit,

$$
\tan \phi=\rho \sin \lambda /\left(2 \rho_{1} \sin \lambda-\rho \cos \lambda\right)
$$

(2) Let $C D$ be the chord of curvature of the evolute subtending an angle $2 \lambda$ at its centre $E$; then

$$
\tan \phi=\frac{Q N}{C D-C N}=\frac{Q N}{N D}=\tan Q D N \text {. }
$$

Hence $\phi=Q D N$; that is, the normal at $Q$ passes through $D$ as in case II.

(3) Also

$$
\left(\mathrm{QQ} Q^{\prime}\right)^{2}=(\mathrm{Q} q)^{2}+\left(\mathrm{Q}^{\prime} q\right)^{2}
$$

$$
\begin{aligned}
\therefore\left(\frac{d s^{\prime}}{d \psi}\right)^{2} & =\rho^{2} \sin ^{2} \lambda+\left(2 \rho_{1} \sin \lambda-\rho \cos \lambda\right)^{2} \\
& =\rho^{2}-4 \rho \rho_{1} \sin \lambda \cos \lambda+4 \rho_{1}{ }^{2} \sin ^{2} \lambda
\end{aligned}
$$

But $\psi+\phi-\psi^{\prime}=\lambda$ and therefore $\frac{d \psi^{\prime}}{d \psi}=1+\frac{d \phi}{d \psi}$

$$
\therefore \rho^{\prime} \equiv \frac{d s^{\prime}}{d \psi^{\prime}}=\frac{\left.\left(\rho^{2}+4 \rho_{1}{ }^{2} \sin ^{2} \lambda-4 \rho \rho_{2} \sin \lambda \cos \lambda\right)\right)^{2}}{\rho^{2}-4 \rho \rho_{1} \sin \lambda \cos \lambda+6 \rho_{1} \sin ^{2} \lambda-2 \rho \rho_{2} \sin ^{2} \lambda}
$$

after reduction.

(4) Again $\tan \phi=\rho \sin \lambda /\left(2 \rho_{1} \sin \lambda-\rho \cos \lambda\right)$

may be written in the form

$$
\left(2 \rho_{1} \sin \lambda-\rho \cos \lambda\right) \sin \phi-\rho \sin \lambda \cos \phi=0
$$

Differentiating with respect to $\psi$, we have

$\left\{\left(2 \rho_{2} \sin \lambda-\rho \cos \lambda\right) \sin \phi-\rho_{1} \sin \lambda \cos \phi\right\}+$

$$
\left\{\left(2 \rho_{1} \sin \lambda-\rho \cos \lambda\right) \cos \phi+\rho \sin \lambda \sin \phi\right\} \frac{d \phi}{d \psi}=0
$$


this leads to the geometrical construction in Figure 20 where EF is the chord of curvature of the second evolute subtending an angle $2 \lambda$ at its centre. Hence as in II. we find

$$
\begin{aligned}
& \text { (i) } \frac{d \phi}{d \psi}=-\frac{\mathrm{DH}}{\mathbf{Q D}}, \\
& \text { (ii) } \frac{d \psi^{\prime}}{d \psi}=\frac{\mathbf{T H}}{\mathbf{T ~ D}},
\end{aligned}
$$

and

(iii) $\rho^{\prime}=Q^{2} / \mathrm{TH}$.

IV.

If in II. or III. the tangent at $Q$ be drawn to the circle of curvature, the envelope of this tangent is closely connected with the evolute of $P$.

(1) If $\mathrm{R}$ (Fig. 21) is the intersection of consecutive tangents $R Q^{\prime} Q N$ is a circle, and therefore $\angle Q R N$ is ultimately equal to $\phi=\angle Q D N$. Thus QNDR is cyclic and - QRD is a right angle. Hence the envelope touches the tangent at the foot of the perpendicular from $D$, where $C D$ is the chord of curvature of the evolute subtending the angle $2 \lambda$ at the centre. Also

$$
\mathrm{QR}=\mathrm{CD} \sin \lambda=2 \rho_{1} \sin ^{2} \lambda .
$$

(2) If $R^{\prime}$ be a neighbouring point on this envelope

$$
\begin{gathered}
\mathbf{R} \mathbf{R}^{\prime}=-\mathbf{Q} Q^{\prime} \cos (\phi+\lambda)+\mathbf{Q}^{\prime} \mathbf{R}^{\prime}-\mathbf{Q R} \\
=-\mathbf{Q} \mathbf{Q}^{\prime}(\cos \phi \cos \lambda-\sin \phi \sin \lambda)+2\left(\rho_{1}+\delta \rho_{1}\right) \sin ^{2} \lambda-2 \rho_{1} \sin ^{2} \lambda \\
=-\cos \lambda\left(2 \rho_{1} \sin \lambda-\rho \cos \lambda\right) \delta \psi+\rho \sin ^{2} \lambda \delta \psi+2 \delta \rho_{1} \sin ^{2} \lambda \\
\quad \therefore \rho^{\prime}=\frac{d s^{\prime}}{d \psi^{\prime}}=2 \rho_{2} \sin ^{2} \lambda+\rho-2 \rho_{2} \sin \lambda \cos \lambda,
\end{gathered}
$$

since $\psi^{\prime}-\psi=\pi-2 \lambda$ and therefore $\delta \psi^{\prime}=\delta \psi$.

(3) If EF be the chord of the second evolute as in III., its projection is $D G=2 \rho_{2} \sin ^{2} \lambda$, and $R D=$ sum of projections of $\mathrm{CQ}_{1} \mathrm{CD}=\rho-2 \rho_{1} \sin \lambda \cos \lambda$. Hence $\mathrm{RG}=\rho^{\prime}$, that is the centre of curvature of the envelope is the projection of $F$ on the normal at $R$. 
V.

If on the tangent at $P$ a length $P Q$ equal to the radius of curvature be measured, the locus of $Q$ has the following properties.

By projection we have (Fig. 22)

$$
\begin{aligned}
\tan \phi & =\coprod_{1} \rho d \psi /\left(\mathbf{P Q}+\mathbf{P P}^{\prime}-\mathbf{P}^{\prime} \mathbf{Q}^{\prime}\right)=L \rho \delta \psi /(\delta s-\delta \rho) \\
& =\rho /\left(\rho-\rho_{1}\right)=\tan \mathrm{EPQ},
\end{aligned}
$$

if $\mathrm{E}$ be the centre of curvature of the evolute. Hence the tangent at $Q$ is the reflection of $E Q$ in the tangent at $P$.

Again

$$
\left(Q^{\prime}\right)^{2}=\rho^{2} \delta \psi^{2}+(\delta s-\delta \rho)^{2}
$$

and therefore $\frac{d s^{\prime}}{d \psi^{\prime}}=\mathrm{EQ} . \quad$ But $\psi^{\prime}=\psi-\phi$,

whence $\quad \rho^{\prime}=\frac{d s^{\prime}}{d \psi^{\prime}}=\frac{d s^{\prime}}{d \psi} / \frac{d \psi^{\prime}}{d \psi}=\frac{\left\{\rho^{2}+(\rho-\rho)^{2}\right\}}{2 \rho^{2}-2 \rho \rho_{1}+2 \rho_{1}^{2}-\rho \rho_{2}}$

If a length be measured on the opposite side of the tangent, we find $\tan \phi=\rho /\left(\rho+\rho_{1}\right)$, etc.

For any length $\kappa \rho$ measured along the tangent, similar results may be established with slight modifications. 